\title{
Charakterisierung von Intermetallischen Phasen bei Verzinkten Hochfesten Stählen Mittels Brom-Ätzung
}

\author{
Magdalena Maderthaner ${ }^{1,2}$, Alexander Jarosik ${ }^{1}$ und Roland Haubner ${ }^{2}$ \\ ${ }^{1}$ voestalpine Stahl GmbH, Linz, Österreich \\ ${ }^{2}$ Institut für Chemische Technologien und Analytik, Technische Universität Wien, Wien, Österreich
}

Eingegangen 8. Februar 2019; angenommen 12. März 2019; online publiziert 27. März 2019

Zusammenfassung: Die Ätzung mit Brom eignet sich zum Freilegen von Oberflächen- und Korngrenzenoxiden, dem Auffinden von FeZn-Phasen sowie zur Gefügebestimmung. $\mathrm{FeZn}$-Phasen werden ebenso wenig wie $\mathrm{Fe}_{2} \mathrm{Al}_{5-x} \mathrm{Zn}_{\mathrm{x}}-\mathrm{Kristal}-$ le und Oxide von Brom angegriffen. Durch die Brom-Ätzung ist es möglich, alle bei der Feuerverzinkung auftretenden Phänomene gleichzeitig metallographisch zu begutachten.

Beim Glühen von speziellen Stählen in trockener Atmosphäre (14 ppm $\mathrm{H}_{2} \mathrm{O}$ ) können sich an der Stahloberfläche Si-reiche Oxide bilden, die ein Wachstum von $\mathrm{Fe}_{2} \mathrm{Al}_{5-\mathrm{x}} \mathrm{Zn}_{\mathrm{x}^{-}}$ Kristallen, welche als Hemmschicht dienen, stellenweise verhindern. Hier kann es zum Wachstum von FeZn-Phasen kommen. Glüht man bei einem höheren Wasserdampfgehalt von 380 ppm, kann man mittels SEM (Scanning Electron Microscope) eine randentkohlte Zone im Stahl feststellen. Dieses Ergebnis stimmt überein mit GDOES-Tiefenprofilen (Glow Discharge Optical Emission Spectroscopy). Oxide entlang der Korngrenzen sind Mischoxide, intrakristalline Oxide (hier ist die Sauerstoffkonzentration geringer als an der Korngrenze) konnten mittels EDX (Energy Dispersive X-Ray Spectroscopy) als $\mathrm{SiO}_{2}$ identifiziert werden.

\section{Schlüsselwörter: Feuerverzinken, Selektive Oxidation}

Characterization of Intermetallic Phases on Hot-dip Galvanized High-strength Steels by Means of Bromine Etching

Abstract: The etching with bromine is suitable for the exposure of intermetallic phases as $\mathrm{Fe}_{2} \mathrm{Al}_{5-x} \mathrm{Zn}_{\mathrm{x}}$-crystals and FeZn-phases and for the microstructural determination. FeZn phases as well as $\mathrm{Fe}_{2} \mathrm{Al}_{5-\mathrm{x}} \mathrm{Zn}_{\mathrm{x}}$-crystals and oxides are not attacked by bromine. The bromine etching makes it possible to examine all phenomena occurring during hotdip galvanizing at the same time metallographically.

M. Maderthaner ( $\triangle)$

voestalpine Stahl $\mathrm{GmbH}$,

voestalpine-Straße 3,

4020 Linz, Österreich

magdalena.maderthaner@voestalpine.com
When high strength steels are annealed in a dry atmosphere ( $14 \mathrm{ppm} \mathrm{H}_{2} \mathrm{O}$ ), Si-rich oxides can be formed on the steel surface, which prevent the growth of $\mathrm{Fe}_{2} \mathrm{Al}_{5-\mathrm{x}} \mathrm{Zn}_{\mathrm{x}}$-crystals serving as an inhibiting layer. This can lead to the undesired growth of FeZn phases. Annealing at a higher water vapor content of $380 \mathrm{ppm}$ leads to an decarburized zone at the surface of the steel, which can be detected by means of SEM (Scanning Electron Microscope). This result is consistent with GDOES depth profiles (Glow Discharge Optical Emission Spectroscopy). Oxides along the grain boundaries are mixed oxides; intracrystalline oxides (here the oxygen concentration is lower than at the grain boundary) could be identified by means of EDX (Energy Dispersive X-Ray Spectroscopy) as $\mathrm{SiO}_{2}$.

Keywords: Hot-dip galvanizing, Selective Oxidation

\section{Einleitung}

Hochfeste Stahlbleche zeichnen sich durch ihre hohe spezifische Festigkeit bei gleichzeitig guter Umformbarkeit für den Automobilen Leichtbau aus. Um den Stahl vor Korrosion zu schützen und zu dessen Langlebigkeit beizutragen, wird auf das Stahlband eine Zinkschicht von wenigen $\mu \mathrm{m}$, typischerweise im kontinuierlichen Feuerverzinkungsprozess, aufgebracht. Diese Zinkschicht wirkt in der Folge sowohl als schützende Barriere als auch im Falle von Verletzungen der Schicht als Opferanode.

Bei der Herstellung wird das kaltgewalzte Stahlband zuerst interkritisch geglüht. Diese Glühbedingungen sind im Allgemeinen reduzierend für Eisen, jedoch oxidierend für die unedlen Legierungselemente Si und Mn. Infolgedessen werden diese sauerstoffaffinen Elemente selektiv oxidiert [1]. In der Folge taucht das Band bei ca. $460^{\circ} \mathrm{C}$ in die flüssige $\mathrm{Zn}(\mathrm{Al}, \mathrm{Fe})$-Schmelze ein und die Stahlbandoberfläche reagiert in Form einer reaktiven Benetzung mit dem $\mathrm{Zn}(\mathrm{Al}$, Fe)-Bad unter Bildung einer flächendeckenden Schicht aus $\mathrm{Fe}_{2} \mathrm{Al}_{5-\mathrm{x}} \mathrm{Zn}_{\mathrm{x}}-$ Kristallen. Wenn sich diese sogenannte Hemmschicht nicht gut ausbildet, kann es zum Wachstum von 
spröden, intermetallischen FeZn-Phasen kommen. Diese Phasen sind unerwünscht, da sie die Oberflächenqualität des verzinkten Stahlbandes verschlechtern und zu ZnHaftungsproblemen führen können [2].

\section{Experimentelles}

Für diese Arbeit wurde ein im Labor hergestellter Stahl mit etwa $1,5 \mathrm{wt} \% \mathrm{Si}, 2,5 \mathrm{wt} \% \mathrm{Mn}, 0,2 \mathrm{wt} \% \mathrm{C}$ verwendet. Dieses Stahlblech wurde auf die Probendimension von $200 \mathrm{~mm} \times 130 \mathrm{~mm}$ geschnitten und bei $80^{\circ} \mathrm{C}$ in einer alkalischen Lösung gereinigt. Die Proben wurden geglüht und in einem Schmelztauchsimulator feuerverzinkt.

Die Probentemperatur wurde mit Thermoelementen aufgezeichnet. Die Proben wurden für $60 \mathrm{~s}$ bei $840^{\circ} \mathrm{C}$ in $\mathrm{N}_{2}$ mit $5 \% \mathrm{H}_{2}$ geglüht. Die Kühlung erfolgt mit $\mathrm{N}_{2}$ mit $1 \% \mathrm{H}_{2}$ mit $25^{\circ} \mathrm{C} / \mathrm{s}$, dann wurde die Temperatur für $3 \mathrm{~s}$ auf $460^{\circ} \mathrm{C}$ gehalten (um das Eintauchen ins Zn-Bad zu simulieren bzw. tatsächlich einzutauchen), anschließend wurde mit etwa $10^{\circ} \mathrm{C} / \mathrm{s}$ weiter abgekühlt (siehe Abb. 1). Der Wasserdampfgehalt in der Glühatmosphäre wurde auf 14 ppm (Taupunkt $-60^{\circ} \mathrm{C}$ ) bzw. $380 \mathrm{ppm}$ (Taupunkt $-30^{\circ} \mathrm{C}$ ) eingestellt. Der Taupunkt wurde mit einem 4-stufigen Tauspiegelhygrometer (Modell 1311 DR von General Eastern) bestimmt.

Die geglühten Proben wurden mit einer Schlagschere zerschnitten, in Bakelit heißeingebettet und geschliffen mit den Körnungen 180, 320, 800 und 1200. Anschließend wurden die Querschliffe poliert mit einem Mol-Tuch mit $3 \mu \mathrm{m}$ Diamant-Suspension und mit einem Dur-Tuch mit $1 \mu \mathrm{m}$ Diamant-Suspension. Abschließend wurden sie mit in absolutem Ethanol gelöstem Brom geätzt [3]. Am Schluss wurde die alkoholische Brom-Lösung mit $\mathrm{Na}_{2} \mathrm{~S}_{2} \mathrm{O}_{3}$-Lösung neutralisiert.

Die geätzten Querschliffe wurden mit Gold besputtert und einem Cu-Band kontaktiert. Die SEM-Aufnahmen (Rasterelektronenmikroskop) wurden mit einem supra35 (Carl Zeiss) mit einem EDX-System (Oxford Instruments, Detektorgröße $80 \mathrm{~mm}^{2}$ ) durchgeführt. Eine hohe Oberflächensensivität erreichte man, indem mit einer niedrigen Beschleunigungsspannung von $2 \mathrm{keV}$ gearbeitet wurde. EDX wurden mit $10 \mathrm{keV}$ durchgeführt, da ein Elektronen- strahl mit einer höheren Beschleunigungsspannung die filigranen Oxidstrukturen zerstören kann.

Die GDOES-Tiefenprofile wurden mit einem GDA 750 (Spectruma Analytik) aufgenommen, kalibiert wurde gemäß DIN EN ISO 10012. Es wurde für 300 s gesputtert, dabei wurde eine Tiefe von $4 \mu \mathrm{m}$ erreicht. Die Sputterrate liegt bei etwa $15 \mathrm{~nm} / \mathrm{s}$. Die gesputterte Probenfläche ist ringförmig mit einem Durchmesser von $4 \mathrm{~mm}$. In der Probenkammer herrscht eine Ar-Atmosphäre mit einem Druck von 300 Pa.

\section{Ergebnisse}

Die Ätzung mit Brom eignet sich zum Auffinden von FeZnPhasen sowie zur Gefügebestimmung. Abb. 2 gibt einen Überblick über den Informationsgehalt einer mit Brom geätzten verzinkten Stahlprobe.

Die Br-geätzten Proben weisen große Ähnlichkeit mit elektropolierten Proben auf. Zn ist die unedelste Komponente im System und wird deswegen bevorzugt abgetragen. Daher eignen sich besonders verzinkte Proben für die Br-Ätzung, weil die Zn-Schicht als Abstandhalter wirkt, sodass die Oberfläche nach der Ätzung gut betrachtet werden kann.

Säulenförmige FeZn-Phasen bleiben ebenso stehen wie $\mathrm{Fe}_{2} \mathrm{Al}_{5-\mathrm{x}} \mathrm{Zn}_{\mathrm{x}}-\mathrm{Hemmschicht-Kristalle.} \mathrm{Auch} \mathrm{das} \mathrm{Gefüge} \mathrm{wird}$ je nach Kohlenstoffgehalt unterschiedlich angeätzt.

\subsection{Metallische Phasen - Gefüge}

Je weniger Kohlenstoff eine Phase im Stahl enthält, also je unedler sie ist, desto stärker wird sie angegriffen. Ferrit wird stark abgetragen und sieht glatt aus. Durch oberflächliche Entkohlung bildet sich an der Bandoberfläche ein Saum aus Ferrit (Randferrit). Martensit, angelassener Martensit und nadelförmiger Bainit lassen sich aufgrund ihres charakteristischen Aussehens unterscheiden.

Das Br-geätzte Gefüge sieht ähnlich aus wie nach dem Elektropolieren oder einer Nital-Ätzung.

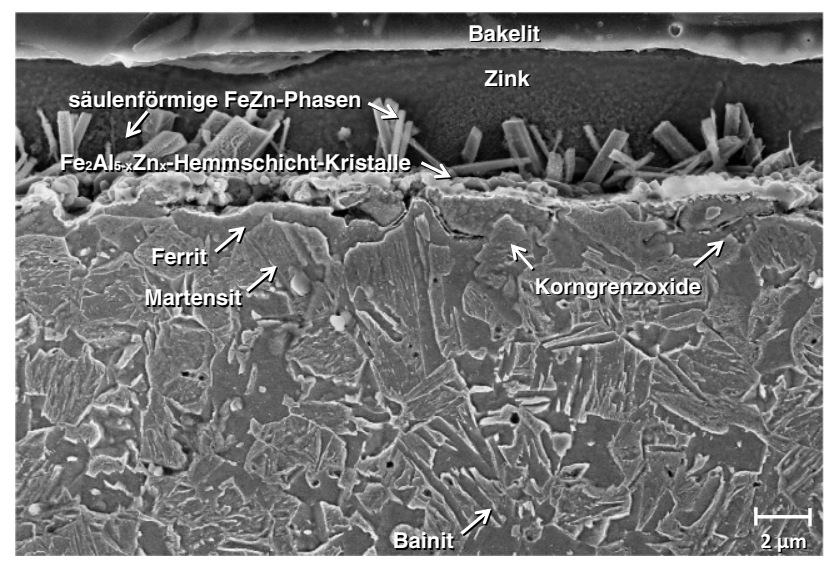

Abb. 2: SEM-Querschliff eines Brom-geätzten verzinkten Stahlbands

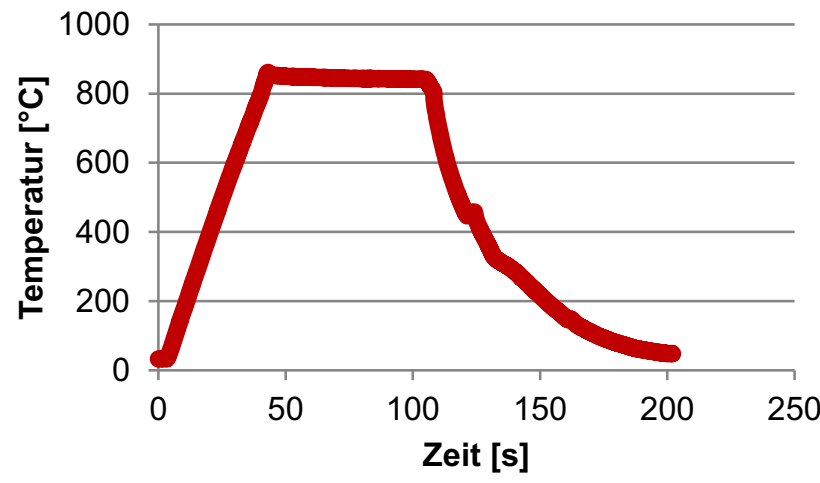

Abb. 1: Temperaturbereich der Proben im Schmelztauchsimulator 
Abb. 3: SEM-Querschliff eines geglühten Stahls mit $380 \mathrm{ppm}$ $\mathrm{H}_{2} \mathrm{O}$ in der Glühatmosphäre. Ein $1 \mu \mathrm{m}$ breiter Saum Randferritbildung ist erkennbar (a), das korrespondierende Kohlenstoff-GDOES-Tiefenprofil (b)

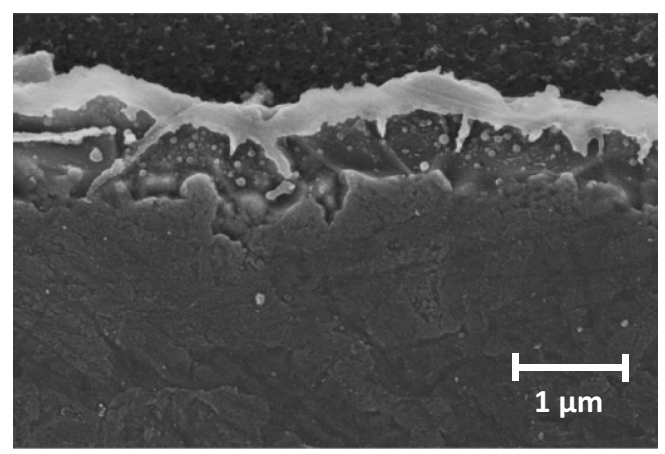

a

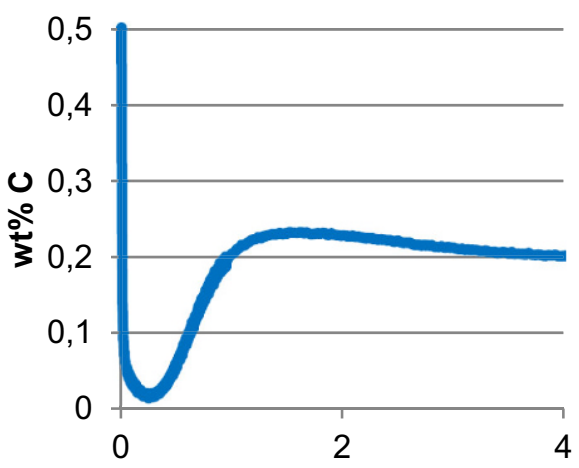

Tiefe $[\mu \mathrm{m}]$

\subsection{Metallische Phasen - Randferrit}

Atmosphärisches $\mathrm{H}_{2} \mathrm{O}$ reagiert beim Glühen mit $\mathrm{C}$ aus dem Stahl zu CO. Das führt zu einer Randentkohlung, wodurch zu wenig $C$ in den Randbereichen gelöst ist, um Bainit oder Martensit zu bilden, sodass sich Ferrit bildet. Eine Randentkohlung findet nur bei ausreichend $\mathrm{H}_{2} \mathrm{O}$ in der Glühatmo-

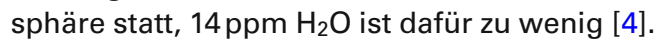

In Abb. 3 ist ein gleichmäßiger Saum von Randferrit zu sehen. Die Daten decken sich sehr gut mit GD-OES-Tiefenprofilen von demselben Stahl im geglühten Zustand. In einer Tiefe von $0,5 \mu \mathrm{m}$ wird ein Minimum von $0,02 \mathrm{wt} \% \mathrm{C}$ gemessen, nach $1,5 \mu \mathrm{m}$ erreicht er wieder den Soll-Wert von $0,2 \mathrm{wt} \% \mathrm{C}$.

\subsection{Metallische Phasen - Intermetallische- Phasen}

Das Vorliegen einer deckenden intermetallischen $\mathrm{Fe}_{2} \mathrm{Al}_{5-x} \mathrm{Zn}_{\mathrm{x}}-\mathrm{Hemmschicht}$, die aus einzelnen Kristallen besteht, korrespondiert mit einer guten Zn-Haftung. Die $\mathrm{Fe}_{2} \mathrm{Al}_{5-\mathrm{x}} \mathrm{Zn}_{\mathrm{x}}-\mathrm{Kristalle}$ lassen sich deutlich erkennen. Diese Hemmschicht verhindert das Wachstum spröder FeZnPhasen.

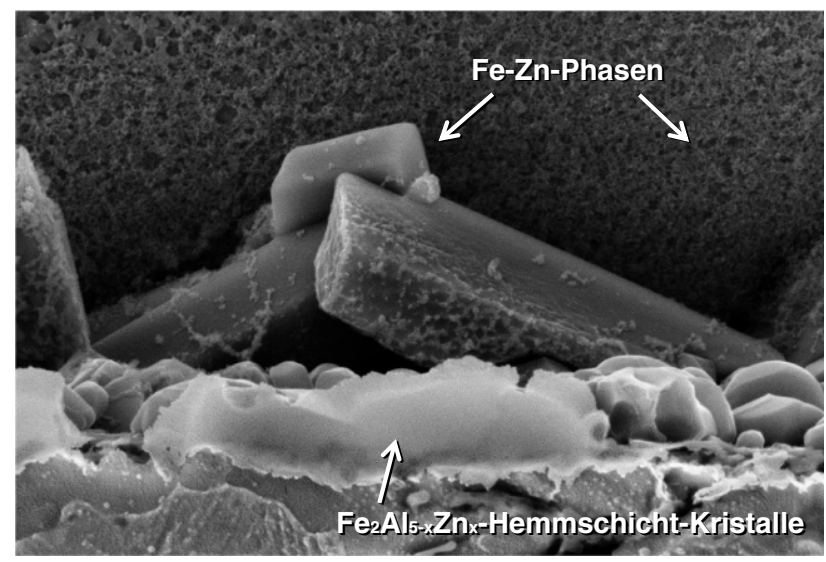

Abb. 4: Säulenartige FeZn-Phasen und Fe2Al5-xZn $n_{x}-$ Kristalle sind eindeutig unterscheidbar
Intermetallische FeZn-Phasen können auftreten, wenn die $\mathrm{Fe}_{2} \mathrm{Al}_{5-\mathrm{x}} \mathrm{Zn}_{\mathrm{x}}-\mathrm{Hemmschicht}$ die Stahloberfläche nicht ausreichend bedeckt. Schnellwachsende Säulen- oder Plattenartige FeZn-Phasen können die Zn-Schicht durchstoßen. Dies geht im Allgemeinen einher mit einer schlechten ZnHaftung und schlechter Oberflächenqualität. Daher ist die Detektion von FeZn-Phasen hilfreich, um Aussagen über die Qualität der Zn-Schicht treffen zu können.

In Abb. 4 lassen sich die beiden intermetallischen Phasen sowohl durch ihre charakteristische Morphologie als auch EDX eindeutig unterscheiden.

\section{Zusammenfassung}

Die Brom-Ätzung ist gut geeignet, um Gefüge, intermetallische Phasen und Oxide von verzinkten Stahlblechen hervorzuheben. Besonders die gleichzeitige Darstellung von FeZn-Phasen und $\mathrm{Fe}_{2} \mathrm{Al}_{5-\mathrm{x}} \mathrm{Zn}_{\mathrm{x}}-\mathrm{Hemmschichtkristallen} \mathrm{ist} \mathrm{be-}$ merkenswert. Ferrit, Martensit, angelassener Martensit und Bainit lassen sich aufgrund ihres charakteristischen Aussehens gut unterscheiden.

Ein Vorteil der Br-Ätzung ist auch, dass es zu keiner Gasentwicklung kommt, die ansonsten zerbrechliche Strukturen wegspülen könnte.

Beim höheren Wasserdampfgehalt in der Glühatmosphäre $\left(380 \mathrm{ppm} \mathrm{H}_{2} \mathrm{O}\right)$ ist ein $1 \mu \mathrm{m}$ dicker Saum aus Randferrit an der Stahloberfläche zu erkennen. Beim niedrigeren Taupunkt ( $14 \mathrm{ppm} \mathrm{H}_{2} \mathrm{O}$ ) gibt es keinen Randferrit, da zu wenig Wasser in der Atmosphäre ist um mit dem Kohlenstoff aus dem Stahl zu CO zu reagieren.

Funding. Open access funding provided by TU Wien (TUW).

Open Access Dieser Artikel wird unter der Creative Commons Namensnennung 4.0 International Lizenz (http://creativecommons.org/licenses/ by/4.0/deed.de) veröffentlicht, welche die Nutzung, Vervielfältigung,

Bearbeitung, Verbreitung und Wiedergabe in jeglichem Medium und Format erlaubt, sofern Sie den/die ursprünglichen Autor(en) und die Quelle ordnungsgemäß nennen, einen Link zur Creative Commons Lizenz beifügen und angeben, ob Änderungen vorgenommen wurden.

Hinweis des Verlags. Der Verlag bleibt in Hinblick auf geografische Zuordnungen und Gebietsbezeichnungen in veröffentlichten Karten und Institutsadressen neutral. 


\section{Literatur}

1. Maderthaner, M.; Jarosik, A.; Angeli, G.; Haubner, R.: Effect of dew point on the selective oxidation of advanced high strength steels, Materials Science Forum, 891 (2017), pp 292-297

2. Marder, A.R.: The metallurgy of zinc-coated steel, Progress in Materials Science, 45 (2000), p 191

3. Miyama, E.; Voitit, C.; Pohl, M.: Zementitnachweis zur Unterscheidung von Bainitstufen in modernen, niedriglegierten Mehrphasenstählen, Practical Metallography, 48 (2011), p 261

4. Maderthaner, M.; Jarosik, A.; Angeli, G.; Haubner, R.: 3rd Generation Hot-Dip Galvanized Steel Sheet for Automobile Manufacturing Interface Reactions between Zinc and Metal Oxide, Key Engineering Materials, 742 (2017), pp 463-470 